\title{
An integro-differential equation with variable delay arising in machine tool vibration
}

\author{
Feng-Bin Wang ${ }^{1,2}$, Stephen A. Gourley ${ }^{3}$ and Yanyu Xiao ${ }^{4}$ \\ ${ }^{1}$ Department of Natural Science in the Center for General Education, Chang Gung \\ University, Guishan, Taoyuan 333, Taiwan. \\ ${ }^{2}$ Community Medicine Research Center, Chang Gung Memorial Hospital, Keelung, \\ Keelung 204, Taiwan. \\ ${ }^{3}$ Department of Mathematics, University of Surrey, Guildford, Surrey, GU2 7XH, UK. \\ ${ }^{4}$ Department of Mathematical Sciences, McMicken College of Arts \& Sciences, \\ University of Cincinnati, Cincinnati, OH 45221-0025, USA.
}

\begin{abstract}
We rigorously derive an integro-differential equation as a model for the possible onset of regenerative chatter during a turning process using a lathe. The cut is made parallel to the axis of rotation of the spindle. The model allows the spindle speed to continuously vary with time, which results in the presence of a variable time delay determined from a threshold condition. We present a number of conditions sufficient for the elimination of chatter, these emphasize sufficiently low feed rate or sufficiently high spindle speed. Numerical simulations cast light on the effect of a sinusoidally varying spindle speed, a feature of some modern lathes. Spindle speed variation can cure chatter but does not necessarily do so, and can fail at higher values of the tool feed rate.
\end{abstract}

Mathematics Subject Classifications (2010): 34K11, 34K20, 34K60, 70J25

Key words: delay differential equation, threshold delay, chatter, spindle speed variation

Short title: Machine tool chatter and variable spindle speed

\section{Introduction}

This paper is concerned with a delay differential equation that arises in the modelling of vibration (chatter) that can occur when machining a rotating workpiece using a lathe. We consider the operation of parallel turning, in which the cut is made longitudinally (i.e. the tool is moved parallel to the axis of the spindle). Cutting processes in general can yield unacceptable surface quality, where vibrations result in waves on the finished surface, and it affects processes including turning using a lathe (as in this paper), milling, boring and grinding. In the case of a turning process, measures that can help to eliminate vibrations include rigidifying the workpiece as much as possible, careful choice of the spindle speed, 
and the use of a modern lathe that can continuously vary the spindle speed. Machine tool chatter in general has been fairly widely studied since the 1950s, though many of the scenarios under consideration lead to equations similar to (1.1) and do not extend to the scenario of the present paper, namely, parallel turning with vibrations perpendicular to the spindle axis. This leads to an integro-differential equation. Previous studies of chatter in various engineering contexts are numerous and include the book by Tobias [23] and, in more recent years, Nigm [11], Shi and Tobias [18], Park and Qin [16] and Hős and Champneys [6]. A readable introduction can be found in Chapter 6 of Erneux [5]. The book by Stone [21] provides extensive discussion of engineering issues and detailed mathematical modelling for cases with constant time delays.

In contrast to the situation under consideration in the present paper (see Fig. 1), a more well studied equation arises in the context of a facing process, where the tool is moved towards, and perpendicular to, the spindle axis of the lathe. That scenario gives rise to a differential equation the linearised form of which can be put into the form

$$
m \frac{d^{2} y(t)}{d t^{2}}+c \frac{d y(t)}{d t}+k y(t)+F_{c}^{\prime}\left(d_{0}\right)(y(t)-y(t-\tau))=0
$$

where $y(t)$ at time $t$ is the deviation of the tool from its desired position due to vibration, and the last term is the linearisation of the cutting force. Experiments show a nonlinear relationship, in the form of a simple power law with exponent less than 1 , between the cutting force $F_{c}(d)$ on the tool and the chip thickness $d$, and the quantity $d_{0}$ in (1.1) is the desired steady state chip thickness. If $\Omega$, the angular velocity of the spindle in radians per second, is constant then the delay $\tau$ in (1.1) is given by $\tau=2 \pi / \Omega$. If the spindle speed varies with time then the delay $\tau$ becomes time-dependent, a significant complication. Delays are often the cause of instability; this is well known not only in engineering but also in ecological and epidemiological contexts (see, for example, the books by Bellman and Cooke [2], Cushing [4], Erneux [5], Kuang [10], Stone [21] or Wu [25]). Chatter can often be eliminated by increasing, rather than reducing, the spindle speed since increasing it lowers the delay $\tau$.

Equation (1.1) can be used to predict the onset of undamped vibrations of the cutting tool that cause the finished workpiece to have a wavy surface. An equation having the exact form of (1.1) except that $\tau$ in (1.1) is replaced by a periodic $\tau(t)$, to allow for periodic variation in spindle speed, appears as equation (18) in Insperger and Stépán [7]. In that paper the emphasis is on the use of a technique called the semidiscretization method to numerically compute the stability boundaries, a challenging task in the case of an equation with variable time delay. Their analysis showed that varying the spindle speed can make it possible to increase critical depths of cut at lower spindle speeds but they found no improvement at higher speeds. Seguy et al [17] apply the semidiscretization method to the stability problem for the case of variable speeds in milling, which is more complex than in turning. A nonlinear version of (1.1), derived from a Taylor expansion of the cutting force retaining terms up to third order, rather than just the linear term as in (1.1), has been considered by Kalmár-Nagy et al [8] who went beyond linearised analysis to present a rigorous investigation of the Hopf bifurcation with the aid of center manifold theory. Wahi and Chatterjee [24] modelled, using a similar equation, a turning process (with constant spindle speed, and therefore constant delay) in the case 


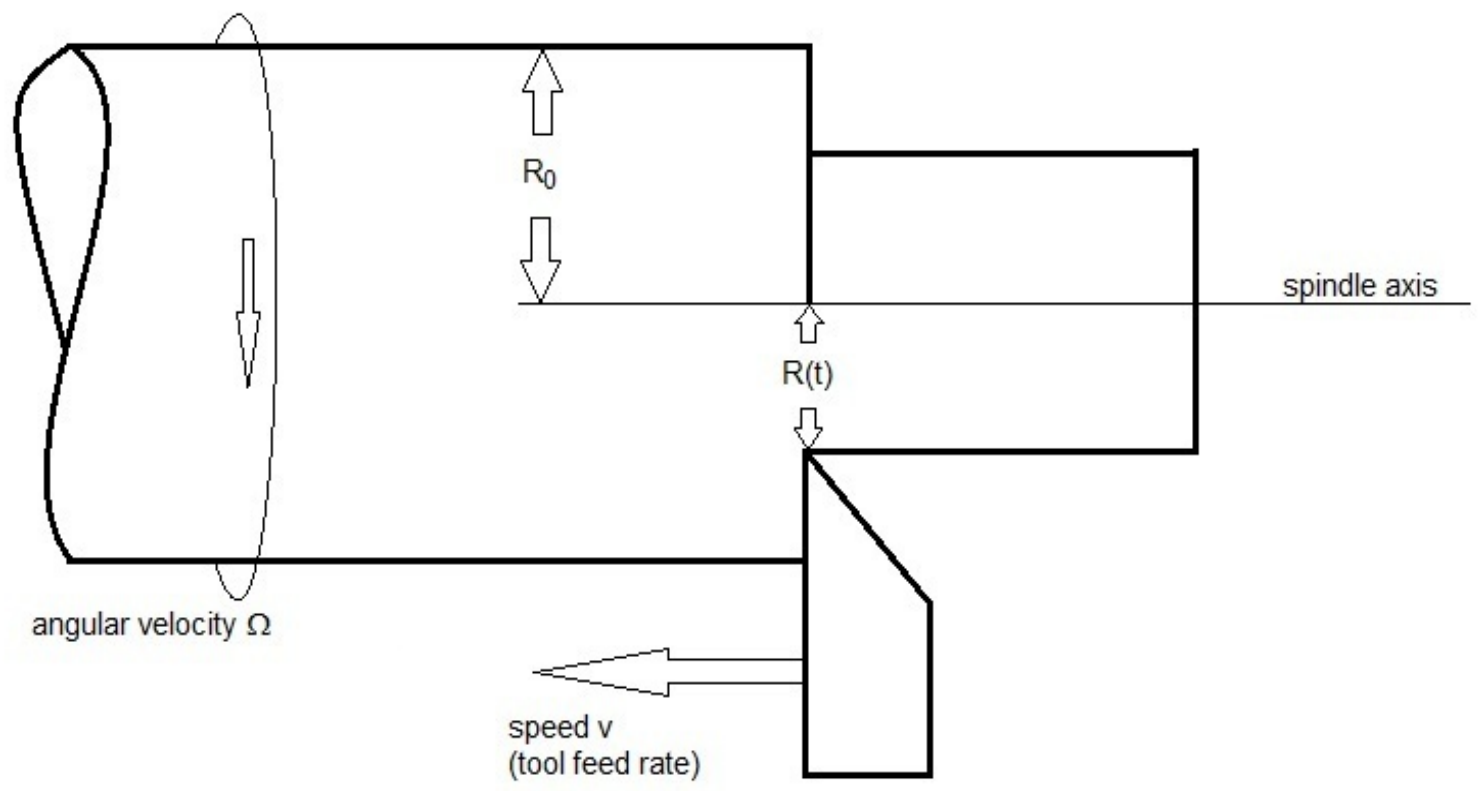

Figure 1: The experimental set-up modelled by equation (2.6). The tool moves parallel to the spindle axis with fixed speed $v$, and all vibration of the tool is in the radial $R(t)$ direction.

when the tool is moved parallel to the spindle axis, as in the present paper. However, their delay differential equation is different from our integro-differential equation (2.6) because they were concerned with vibrations parallel to the spindle axis, whereas in the present paper we only consider vibrations perpendicular to the spindle axis. Wahi and Chatterjee [24] present a detailed bifurcation study of their nonlinear problems using multiple time scales analysis. A comparable study using this technique is presented in Sri Namachchivaya and Beddini [22]. The present paper pays detailed attention to the derivation of a delay differential equation determining $R(t)$, the radial displacement of the tool tip, in the case of a longitudinal cut (parallel turning) where the spindle speed is allowed to vary continuously with time in a prescribed manner, as shown in Fig. 1. Variation of spindle speed is important because some modern lathes incorporate technology known as spindle speed variation (SSV) that can help to eliminate chatter. Although the cut is parallel to the axis, differently from some previous studies we consider only vibrations perpendicular to the axis. This is important because, in parallel turning, chatter is usually caused primarily by modes of vibration in a plane perpendicular to the axis (Stone [21]). The complication of SSV, and the assumption that vibrations occur in a direction perpendicular to the feed direction, give us a differential equation that no longer has the form of (1.1). Instead, we obtain equation (2.6) which is an integro-differential equation in which the variable delay $\tau(t)$ is determined from a threshold condition. We start by treating the case of a constant spindle speed, presenting two theorems each of 
which yields conditions sufficient for the elimination of chatter. Then we move to the case of a variable spindle speed, presenting a theorem containing conditions sufficient for elimination of chatter that work for any variable spindle speed $\Omega(t)$ and focus more on the role of the tool feed rate $v$. Those conditions are sufficient and not necessary, and do not elucidate in detail the precise effect of varying the spindle speed. That effect is then studied using multiple time scales analysis, the final outcome of which is that varying the spindle speed has the potential to cure high frequency chatter. The results of some numerical simulations are presented that use realistic values for model parameters, and these confirm the predictions.

Previous studies specifically on the effect of spindle speed variation (SSV) include the work of Sri Namachchivaya and Beddini [22], Zhang, Jackson and Ni [26, 27] and Otto and Radons [13] and have focussed on scenarios giving rise to equations having the appearance of (1.1) rather than the integro-differential equation (2.6) of the present paper. SSV gives rise to equations with variable time delays, so $\tau$ in (1.1) would become $\tau(t)$ with $\tau(t)$ a periodic function, since the spindle speed $\Omega(t)$ will be varying in a periodic (sinusoidal, in practice) manner. Otto et al [12] present an efficient numerical approach to the computation of stability diagrams using a simple SSV model, with variable delay, capturing the essentials of both turning and milling. Our equation (2.6) has a variable time delay $\tau(t)$ which is determined from the threshold condition (2.3). Sri Namachchivaya and Beddini [22] use multiple time scales analysis to compute the stability boundary and the bifurcating solutions for their nonlinear model, which incorporates SSV (and therefore variable delays) but models a different scenario yielding an equation more like (1.1) than (2.6).

Before proceeding further, we stress that in this paper we are considering the problem of turning a rigid workpiece with a flexible tool. If the workpiece is thin enough to be flexible, with a tendency to bend away from the tool, then the modes of vibration of the workpiece become as important as those of the tool. This has become a very recent area of research within chatter theory (Otto et al [14], Khasawneh and Otto [9]) which is not explored in the present paper but is briefly mentioned in the concluding remarks.

\section{Model derivation}

We consider the problem of using a lathe to make a longitudinal (i.e. parallel to the axis of rotation of the spindle) cut that aims to reduce the original radius $R_{0}$ of a cylinder of metal to some smaller desired radius $R_{*}$. Vibration may result in a finished workpiece that is not the desired perfect cylinder due to the phenomenon of chatter, as described in the introduction. Of central importance to the mathematical modelling of chatter is the calculation of the forces on the tool, and these depend on the material removal rate (MRR), which is the volume of material removed per unit time.

\subsection{The idealised situation}

It is useful to briefly review the calculation of the MRR in the case when the tool moves such that its distance from the axis of rotation of the workpiece remains constant (no 
vibration), because comparison with this case can provide a useful check when we consider the more complicated case of tool vibration (and, especially, the case of a variable spindle speed). Let $d$ be the depth of the cut, so that $d=R_{0}-R_{*}$ and let $v$ denote the feed rate, which is the linear velocity of the tool as it moves longitudinally. Let $\Omega_{0}$, assumed constant in this subsection, denote the spindle rotation speed in radians per unit time. In the idealised situation that we are presently concerned with, the rotation of the workpiece and longitudinal movement of the tool at a constant velocity $v$ has the effect of removing material from the workpiece in the form of a helix (in practice, small, spiral shaped rings). One can take the view that each revolution removes a ring of material. The time $\tau_{0}$ for one revolution is given by $\tau_{0}=2 \pi / \Omega_{0}$ and in this time the tool moves a distance $v \tau_{0}$ so this is the width of the ring. The ring's depth (difference between outer and inner radii) is $d$. It is not a perfect ring, of course, but one can still take the view that its volume can be calculated by the rotation of a plane area, in the form of a rectangle with dimensions $v \tau_{0}$ and $d$, about the axis of rotation using Pappus's theorem. That theorem yields the volume of the ring of removed material as the area $d v \tau_{0}$ of the rectangle just described, multiplied by the distance travelled by its geometric centroid over one revolution, and this distance is $\pi\left(R_{0}+R_{*}\right)$. So the volume is $\pi\left(R_{0}+R_{*}\right) d v \tau_{0}$. The number of revolutions per unit time is $1 / \tau_{0}$ and thus the MRR, in this idealised situation of no vibration, is given by

$$
\mathrm{MRR}_{\text {ideal }}=\pi\left(R_{0}+R_{*}\right) d v .
$$

This formula, often presented in different notation or in terms of other parameters, is well known.

\subsection{Variable spindle speed and vibration}

We now consider the situation in which $R(t)$, defined as the distance from the tool tip to the axis of rotation at time $t$, is non-constant due to vibration. Ideally, we would wish to have $R(t) \equiv R_{*}$. We will formulate a delay differential equation determining $R(t)$ that has $R_{*}$ as a steady state, which may or may not be stable. At the same time, we also allow the spindle speed to vary, so that the angular velocity of the workpiece is no longer necessarily constant and will be denoted $\Omega(t)$. An important aim is to investigate whether a variable spindle speed can reduce or eliminate the problem of chatter.

Of course, varying the spindle speed also has the effect of varying the time $\tau(t)$ for one revolution, and therefore of the distance travelled by the tool over one revolution (we assume that the tool still moves with a constant velocity $v$ ). We start by calculating $\tau(t)$. Consider a line of points fixed in a particular radius of the rotating cylindrical workpiece, and suppose that at time $t$ those points are aligned in a particular direction and that they were last aligned in that direction one revolution ago at time $t-\tau(t)$. Letting $s$ be some intermediate time, then, if $\theta$ describes the location of the line of points under discussion, and the direction of increasing $\theta$ is the direction of rotation, we have $d \theta=\Omega(s) d s$. Integrating over $s \in[t-\tau(t), t]$ yields

$$
\int_{t-\tau(t)}^{t} \Omega(s) d s=2 \pi
$$

which determines $\tau(t)$. 
Three forces act on the tool. The feed force or thrust force $F_{t}$ is the reaction from the lathe to the force with which the tool is being pushed in the longitudinal (feed) direction. The cutting force $F_{c}$ is in the circumferential direction and is attributable to the cutting action. The radial force $F_{r}$, in the radial direction, tends to push the tool away from the workpiece. We assume that the resultant of these three forces is a function of the material removal rate MRR so that each component, in particular $F_{r}$, is also a function of the MRR. The lack of perfect rigidity of the lathe is accounted for in the modelling by having a spring, with spring constant $k$, tending to push the tool towards the workpiece in the radial direction, and also a damper with damping constant $c$. We assume the force from the spring can be calculated using Hooke's law.

Letting $m$ be the mass of the tool and resolving forces in the radial direction gives

$$
m \frac{d^{2} R(t)}{d t^{2}}+c \frac{d R(t)}{d t}=F_{r}(\mathrm{MRR})-(\text { force from spring }) .
$$

We now calculate the MRR. As noted earlier, each revolution removes a ring-shaped piece of material though the characteristics described earlier such as "width" and "depth" of the ring will now vary with time. At time $t$ the cutting edge is distant $R(t)$ from the axis but in order to calculate the MRR one has to look at the values of $R(s), s \in[t-\tau(t), t]$ relating to the last revolution. The material being removed at time $t$ has varying depth $R_{0}-R(s), s \in[t-\tau(t), t]$. Consider what happens over a time interval $[s, s+d s]$ with $s \in(t-\tau(t), t)$. Over this time the tool moves a longitudinal distance $v d s$ while the workpiece rotates through an angle $\Omega(s) d s$, and the effect is that the tool removes a volume of material that can be described as the rotation of a plane area (in the form of an infinitesimally thin strip) through an angle. The centroid of the strip is distant $\frac{1}{2}\left(R_{0}+R(s)\right)$ from the axis and therefore, by Pappus's theorem, the volume of material removed in the time interval $[s, s+d s]$ is

$$
\underbrace{\left(R_{0}-R(s)\right) v d s}_{\text {area of strip }} \underbrace{\frac{1}{2}\left(R_{0}+R(s)\right) \Omega(s) d s}_{\text {distance travelled by centroid }}
$$

On cancelling one of the $d s$ 's, one converts the above expression to an instantaneous volume removal rate, but only in respect of the infinitesimal strip under discussion. We then sum over all relevant strips, i.e. sum over all $s \in(t-\tau(t), t)$, to conclude that the material removal rate MRR is given by

$$
\mathrm{MRR}=\frac{1}{2} v \int_{t-\tau(t)}^{t}\left(R_{0}^{2}-R^{2}(s)\right) \Omega(s) d s .
$$

If $R(t)=R_{*}$ is constant then, using (2.3), the above expression becomes $v \pi\left(R_{0}^{2}-R_{*}^{2}\right)$ which is the same as expression (2.2) since we earlier defined $d=R_{0}-R_{*}$ as the depth of cut in the case of constant $R$.

Regarding the lack of perfect rigidity of the setup, which we model by the spring and damper acting on the tool, we let $l_{0}$ be the compression of the spring when the force from the spring is in balance with $F_{r}$ and $R(t) \equiv R_{*}$ is constant, i.e. the lathe is making the desired perfect cut with no vibration. In this situation the force from the spring is $k l_{0}$. If $R$ increases from $R_{*}$ to $R(t)$ then the compression of the spring increases to 
$l_{0}+R(t)-R_{*}$ and the force exerted on the tool by the spring increases to $k\left(l_{0}+R(t)-R_{*}\right)$. The equation of motion, equation (2.4), becomes

$$
m \frac{d^{2} R(t)}{d t^{2}}+c \frac{d R(t)}{d t}=F_{r}\left(\frac{1}{2} v \int_{t-\tau(t)}^{t}\left(R_{0}^{2}-R^{2}(s)\right) \Omega(s) d s\right)-k\left(l_{0}+R(t)-R_{*}\right)
$$

and, in equilibrium, we have $R(t) \equiv R_{*}$ and (using (2.3)),

$$
F_{r}\left(v \pi\left(R_{0}^{2}-R_{*}^{2}\right)\right)=k l_{0}
$$

\section{$3 \quad$ Linearised analysis}

We linearise equation (2.6) about the steady state $R_{*}$ satisfying $(2.7)$ by setting $R(t)=$ $R_{*}+\tilde{R}(t)$, with $\tilde{R}(t)$ small, to obtain the linearised equation for $\tilde{R}(t)$ :

$$
m \frac{d^{2} \tilde{R}(t)}{d t^{2}}+c \frac{d \tilde{R}(t)}{d t}+k \tilde{R}(t)=-v R_{*} F_{r}^{\prime}\left(v \pi\left(R_{0}^{2}-R_{*}^{2}\right)\right) \int_{t-\tau(t)}^{t} \Omega(s) \tilde{R}(s) d s .
$$

We next consider the relatively straightforward case of a constant spindle speed.

\subsection{Constant spindle speed}

If the angular spindle speed is constant, so that $\Omega(t) \equiv \Omega_{0}$, then $\tau(t)$ is also constant and, from (2.3), $\tau(t) \equiv \tau_{0}=2 \pi / \Omega_{0}$. Equation (3.8) becomes

$$
m \frac{d^{2} \tilde{R}(t)}{d t^{2}}+c \frac{d \tilde{R}(t)}{d t}+k \tilde{R}(t)=-v R_{*} F_{r}^{\prime}\left(v \pi\left(R_{0}^{2}-R_{*}^{2}\right)\right) \Omega_{0} \int_{t-\tau_{0}}^{t} \tilde{R}(s) d s
$$

and the ansatz $\tilde{R}(t)=\exp (\lambda t)$ leads to the characteristic equation

$$
m \lambda^{2}+c \lambda+k=-v R_{*} F_{r}^{\prime}\left(v \pi\left(R_{0}^{2}-R_{*}^{2}\right)\right) \Omega_{0}\left(\frac{1-e^{-\lambda \tau_{0}}}{\lambda}\right) .
$$

We prove the following theorem, which provides a set of conditions sufficient to ensure that $\tilde{R}(t) \rightarrow 0$ as $t \rightarrow \infty$. If these conditions hold then we predict there will be no vibration (chatter) in the case of constant spindle speed.

Theorem 1. Suppose the spindle speed is constant, $F_{r}(\cdot)$ has positive derivative, $c \geq$ $\sqrt{2 m k}$ and $2 \pi v R_{*} F_{r}^{\prime}\left(v \pi\left(R_{0}^{2}-R_{*}^{2}\right)\right)<k$. Then all roots $\lambda$ of (3.10) have negative real parts so that, for small perturbations, $R(t) \rightarrow R_{*}$ as $t \rightarrow \infty$ and vibration is eliminated. 
Proof. Suppose, for a contradiction, that there exists a root $\lambda_{0}$ of (3.10) such that $\operatorname{Re} \lambda_{0} \geq 0$. Then

$$
\begin{aligned}
\left|m \lambda_{0}^{2}+c \lambda_{0}+k\right| & =v R_{*} F_{r}^{\prime}\left(v \pi\left(R_{0}^{2}-R_{*}^{2}\right)\right) \Omega_{0}\left|\frac{1-e^{-\lambda_{0} \tau_{0}}}{\lambda_{0}}\right| \\
& =v R_{*} F_{r}^{\prime}\left(v \pi\left(R_{0}^{2}-R_{*}^{2}\right)\right) \Omega_{0}\left|\int_{0}^{\tau_{0}} e^{-\lambda_{0} s} d s\right| \\
& \leq v R_{*} F_{r}^{\prime}\left(v \pi\left(R_{0}^{2}-R_{*}^{2}\right)\right) \Omega_{0} \int_{0}^{\tau_{0}} e^{-\left(\operatorname{Re} \lambda_{0}\right) s} d s \\
& \leq v R_{*} F_{r}^{\prime}\left(v \pi\left(R_{0}^{2}-R_{*}^{2}\right)\right) \Omega_{0} \tau_{0} \\
& =2 \pi v R_{*} F_{r}^{\prime}\left(v \pi\left(R_{0}^{2}-R_{*}^{2}\right)\right) .
\end{aligned}
$$

But, since $\operatorname{Re} \lambda_{0} \geq 0$,

$$
\begin{aligned}
\left|m \lambda_{0}^{2}+c \lambda_{0}+k\right|^{2} & =\left(m \lambda_{0}^{2}+c \lambda_{0}+k\right)\left(m \bar{\lambda}_{0}^{2}+c \bar{\lambda}_{0}+k\right) \\
& =\left(\lambda_{0}+\bar{\lambda}_{0}\right)\left(m c\left|\lambda_{0}\right|^{2}+c k\right)+m\left|\lambda_{0}\right|^{4}+c^{2}\left|\lambda_{0}\right|^{2}+k^{2}+m k\left(\lambda_{0}^{2}+\bar{\lambda}_{0}^{2}\right) \\
& \geq m\left|\lambda_{0}\right|^{4}+c^{2}\left|\lambda_{0}\right|^{2}+k^{2}+m k\left(\lambda_{0}^{2}+\bar{\lambda}_{0}^{2}\right)
\end{aligned}
$$

since $\lambda_{0}+\bar{\lambda}_{0}=2 \operatorname{Re} \lambda_{0} \geq 0$. Letting $\lambda_{0}=a+i b$,

$$
\left|m \lambda_{0}^{2}+c \lambda_{0}+k\right|^{2} \geq m\left(a^{2}+b^{2}\right)^{2}+c^{2}\left(a^{2}+b^{2}\right)+k^{2}+2 m k\left(a^{2}-b^{2}\right) \geq k^{2}
$$

since $c \geq \sqrt{2 m k}$. Thus

$$
k \leq\left|m \lambda_{0}^{2}+c \lambda_{0}+k\right| \leq 2 \pi v R_{*} F_{r}^{\prime}\left(v \pi\left(R_{0}^{2}-R_{*}^{2}\right)\right)
$$

which contradicts the second inequality of the hypotheses.

Theorem 1 yields no insight into the role of the angular spindle speed $\Omega_{0}$. The next theorem provides another sufficient set of conditions for the elimination of vibration; this time by assuring us that it happens for sufficiently large $\Omega_{0}$.

Theorem 2. Suppose the spindle speed is constant, $F_{r}(\cdot)$ has positive derivative and

$$
2 \pi^{2} v R_{*} F_{r}^{\prime}\left(v \pi\left(R_{0}^{2}-R_{*}^{2}\right)\right)<c \Omega_{0} .
$$

Then all roots $\lambda$ of (3.10) have negative real parts so that, for small perturbations, $R(t) \rightarrow$ $R_{*}$ as $t \rightarrow \infty$ and vibration is eliminated.

Proof. The characteristic equation (3.10) is $f(\lambda)=0$ where

$$
f(\lambda)=m \lambda^{2}+c \lambda+k+A \Omega_{0}\left(\frac{1-e^{-\lambda \tau_{0}}}{\lambda}\right), \quad \text { with } A=v R_{*} F_{r}^{\prime}\left(v \pi\left(R_{0}^{2}-R_{*}^{2}\right)\right) .
$$

Letting

$$
g(\lambda)=\frac{1-e^{-\lambda}}{\lambda},
$$

we may further rewrite $f(\lambda)$ in the form

$$
f(\lambda)=m \lambda^{2}+c \lambda+k+A \Omega_{0} \tau_{0} g\left(\lambda \tau_{0}\right)=m \lambda^{2}+c \lambda+k+2 \pi A g\left(\lambda \tau_{0}\right)
$$


using that $\Omega_{0} \tau_{0}=2 \pi$. Since $f(\lambda)$ is analytic, the number of roots of the characteristic equation $f(\lambda)=0$ inside $\operatorname{Re} \lambda>0$ is given by

$$
\frac{1}{2 \pi i} \lim _{R \rightarrow \infty} \int_{\gamma_{R}} \frac{f^{\prime}(\lambda)}{f(\lambda)} d \lambda
$$

where $\gamma_{R}$ denotes a semicircular contour in $\operatorname{Re} \lambda \geq 0$ of radius $R$, with curved part $\gamma_{R}^{\text {curved }}$ and straight part $\gamma_{R}^{\text {straight }}$, defined by

$$
\begin{aligned}
\gamma_{R}^{\text {curved }} & =\left\{\lambda=R e^{i \theta}: \theta \in[-\pi / 2, \pi / 2]\right\} \\
\gamma_{R}^{\text {straight }} & =\{\lambda=i y: y \in[R,-R]\}
\end{aligned}
$$

and the contour is traversed in the anticlockwise sense. To prove the theorem we need to show that formula (3.15) yields an answer of zero (the absence of roots of $f(\lambda)=0$ on the imaginary axis will become clear within the proof).

We shall in fact prove that the number of roots of $f(\lambda)=0$ inside $\operatorname{Re} \lambda>0$ is determined by formula (3.15) to be

$$
1-\frac{1}{\pi}[\arg f(i R)]_{R=0}^{R=\infty}
$$

where the quantity multiplying $1 / \pi$ is the total change in the argument of the complex number $f(i R)$ as $R$ varies from 0 to infinity. We shall show that this total change is $\pi$ when inequality (3.11) holds. Otherwise, it could in principle be any of the alternative values $-\pi,-3 \pi,-5 \pi, \ldots$. In each of these cases the characteristic equation $f(\lambda)=0$ has roots in the right half of the complex plane. All but the first of these latter cases correspond to scenarios in which, as $R$ increases, $f(i R)$ winds around the origin a number of times before setting off to infinity in $\mathbb{C}$. They are excluded by the hypotheses of Theorem 2 .

To prove formula (3.16) we shall first prove that

$$
\frac{1}{2 \pi i} \lim _{R \rightarrow \infty} \int_{\gamma_{R}^{\text {curved }}} \frac{f^{\prime}(\lambda)}{f(\lambda)} d \lambda=1
$$

or, equivalently, that

$$
\lim _{R \rightarrow \infty} \int_{\gamma_{R}^{\text {curved }}}\left(\frac{f^{\prime}(\lambda)}{f(\lambda)}-\frac{2}{\lambda}\right) d \lambda=0
$$

Now

$$
\int_{\gamma_{R}^{\text {curved }}}\left(\frac{f^{\prime}(\lambda)}{f(\lambda)}-\frac{2}{\lambda}\right) d \lambda=\int_{\gamma_{R}^{\text {curved }}} \frac{-c \lambda-2 k+2 \pi A \tau_{0} \lambda g^{\prime}\left(\lambda \tau_{0}\right)-4 \pi A g\left(\lambda \tau_{0}\right)}{\lambda\left(m \lambda^{2}+c \lambda+k+2 \pi A g\left(\lambda \tau_{0}\right)\right)} d \lambda .
$$

Writing this as a sum of four integrals, the next step is to show that each of them approaches zero as $R \rightarrow \infty$. This is routine in the case of those terms not involving the function $g$ in their numerators, and so we restrict attention to those that do. However, for $\operatorname{Re} \lambda \geq 0$,

$$
|g(\lambda)|=\left|\frac{1-e^{-\lambda}}{\lambda}\right|=\left|\int_{0}^{1} e^{-\theta \lambda} d \theta\right| \leq \int_{0}^{1} e^{-\theta \operatorname{Re} \lambda} d \theta \leq 1
$$


and

$$
\left|g^{\prime}(\lambda)\right|=\left|\int_{0}^{1}-\theta e^{-\theta \lambda} d \theta\right| \leq \int_{0}^{1} \theta e^{-\theta \operatorname{Re} \lambda} d \theta \leq \int_{0}^{1} \theta d \theta=\frac{1}{2} .
$$

The term in (3.19) with $g^{\prime}\left(\lambda \tau_{0}\right)$ in its numerator thus approaches zero as $R \rightarrow \infty$ because that term, ignoring its constant coefficient $2 \pi A \tau_{0}$, can be estimated as follows for $R$ sufficiently large. Setting $\lambda=R e^{i \theta}$, and using (3.20) and (3.21),

$$
\begin{aligned}
& \left|\int_{\gamma_{R}^{\text {curved }}} \frac{\lambda g^{\prime}\left(\lambda \tau_{0}\right)}{\lambda\left(m \lambda^{2}+c \lambda+k+2 \pi A g\left(\lambda \tau_{0}\right)\right)} d \lambda\right| \leq \int_{-\pi / 2}^{\pi / 2} \frac{\left|g^{\prime}\left(\tau_{0} R e^{i \theta}\right)\right| R d \theta}{m R^{2}-c R-k-2 \pi A\left|g\left(\tau_{0} R e^{i \theta}\right)\right|} \\
& \leq \frac{\frac{1}{2} R \pi}{m R^{2}-c R-k-2 \pi A} \rightarrow 0 \quad \text { as } R \rightarrow \infty
\end{aligned}
$$

Treatment of the other terms in (3.19) is similar. Thus, (3.17) holds and therefore

$$
\begin{aligned}
& \frac{1}{2 \pi i} \lim _{R \rightarrow \infty} \int_{\gamma_{R}} \frac{f^{\prime}(\lambda)}{f(\lambda)} d \lambda \\
& \quad=1+\frac{1}{2 \pi i} \lim _{R \rightarrow \infty} \int_{\gamma_{R}^{\text {straight }}} \frac{f^{\prime}(\lambda)}{f(\lambda)} d \lambda=1+\frac{1}{2 \pi i} \lim _{R \rightarrow \infty} \int_{R}^{-R} \frac{f^{\prime}(i y)}{f(i y)} i d y \\
& \quad=1+\frac{1}{2 \pi i}[\ln f(-i R)-\ln f(i R)]_{R=0}^{R=\infty} .
\end{aligned}
$$

But $f(i R)$ and $f(-i R)$ are complex conjugates. Thus

$$
\frac{1}{2 \pi i} \lim _{R \rightarrow \infty} \int_{\gamma_{R}} \frac{f^{\prime}(\lambda)}{f(\lambda)} d \lambda=1-\frac{1}{\pi}[\arg f(i R)]_{R=0}^{R=\infty}
$$

and formula (3.16) is proved. To complete the proof of the theorem we must show, using condition (3.11), that

$$
[\arg f(i R)]_{R=0}^{R=\infty}=\pi \text {. }
$$

From (3.13)

$$
g\left(i R \tau_{0}\right)=\frac{\sin R \tau_{0}}{R \tau_{0}}+i \frac{\cos R \tau_{0}-1}{R \tau_{0}}
$$

and therefore, from (3.14),

$$
\begin{aligned}
f(i R) & =-m R^{2}+k+2 \pi A \operatorname{Re} g\left(i R \tau_{0}\right)+i\left(c R+2 \pi A \operatorname{Im} g\left(i R \tau_{0}\right)\right) \\
& =-m R^{2}+k+2 \pi A \frac{\sin R \tau_{0}}{R \tau_{0}}+i\left(c R+2 \pi A \frac{\cos R \tau_{0}-1}{R \tau_{0}}\right) .
\end{aligned}
$$

Moreover, from (3.20), $|g(i R)| \leq 1$ and therefore, for large $R$,

$$
\operatorname{Re} f(i R) \sim-m R^{2}, \quad \operatorname{Im} f(i R) \sim c R
$$


and $f(0)=k+2 \pi A>0$. These facts imply that the total change in the argument of the complex number $f(i R)$, as $R$ goes from 0 to infinity, must be an odd multiple of $\pi$. However, for all $R \in(0, \infty)$, using that $\sin x \leq x$ for all $x \geq 0$,

$$
\begin{aligned}
\operatorname{Im} f(i R) & =c R+2 \pi A \frac{\cos R \tau_{0}-1}{R \tau_{0}}=c R-4 \pi A \frac{\sin ^{2} R \tau_{0} / 2}{R \tau_{0}} \\
& \geq c R-\pi A R \tau_{0}>0
\end{aligned}
$$

because inequality (3.11), the definition of $A$ in (3.12) and the fact that $\Omega_{0} \tau_{0}=2 \pi$, together imply that $c>\pi A \tau_{0}$. The aforementioned facts, with this additional knowledge that in fact $\operatorname{Im} f(i R)>0$ for all $R>0$, imply that the total change in the argument of $f(i R)$ is $\pi$. Therefore, formula (3.16) yields that the characteristic equation $f(\lambda)=0$ has no roots with $\operatorname{Re} \lambda>0$. The last stage of this proof also confirms that $f(\lambda)=0$ has no roots on the imaginary axis. The proof is complete.

\subsection{Variable spindle speed}

For the case of a variable spindle speed we first present the following theorem which guarantees the stability of the steady state $R_{*}$, for a general variable spindle speed $\Omega(t)$, provided a condition is satisfied involving the spring constant $k$, damping $c$, tool mass $m$, initial and desired final radii $R_{0}$ and $R_{*}$, and tool feed rate $v$.

Theorem 3. Suppose that the spindle speed $\Omega(t)$ is periodic, $F_{r}(\cdot)$ has positive derivative and

$$
2 \pi v R_{*} F_{r}^{\prime}\left(v \pi\left(R_{0}^{2}-R_{*}^{2}\right)\right) \int_{0}^{\infty}\left|f_{1}(\eta)\right| d \eta<1
$$

where

$$
f_{1}(t)=\mathcal{L}^{-1}\left\{\frac{1}{m p^{2}+c p+k}\right\},
$$

with $\mathcal{L}$ denoting Laplace transform and $p$ the transform variable. Then, for small perturbations, $R(t) \rightarrow R_{*}$ as $t \rightarrow \infty$ and vibration is eliminated.

Proof. We write the linearised equation (3.8) in the form

$$
m \frac{d^{2} \tilde{R}(t)}{d t^{2}}+c \frac{d \tilde{R}(t)}{d t}+k \tilde{R}(t)=-H(t)
$$

where

$$
H(t)=v R_{*} F_{r}^{\prime}\left(v \pi\left(R_{0}^{2}-R_{*}^{2}\right)\right) \int_{t-\tau(t)}^{t} \Omega(s) \tilde{R}(s) d s .
$$

Applying the Laplace transform $\mathcal{L}$ to (3.25), using $p$ to denote the transform variable, it can be shown in a routine way that the solution of (3.25) for any given $H(t)$ is

$$
\tilde{R}(t)=\mathcal{L}^{-1}\left\{\frac{m p \tilde{R}(0)+m \tilde{R}^{\prime}(0)+c \tilde{R}(0)}{m p^{2}+c p+k}\right\}-\int_{0}^{t} f_{1}(t-s) H(s) d s
$$


where $f_{1}(t)$ is given by (3.24). The first term is an initial transient which decays exponentially to zero as $t \rightarrow \infty$, since it is the inverse Laplace transform of a simple rational function that has two poles both of which have $\operatorname{Re} p<0$. We henceforth ignore that term. Since $H(t)$ is actually given by (3.26), we have effectively converted the problem into an integral equation for $\tilde{R}(t)$ :

$$
\tilde{R}(t)=-v R_{*} F_{r}^{\prime}\left(v \pi\left(R_{0}^{2}-R_{*}^{2}\right)\right) \int_{0}^{t} f_{1}(t-s) \int_{s-\tau(s)}^{s} \Omega(\xi) \tilde{R}(\xi) d \xi d s .
$$

Seeking solutions of the form $\tilde{R}(t)=e^{\lambda t} P(t)$, where $P(t)$ is a periodic function having the same period (denoted $T$ ) as $\Omega(t)$, then making a substitution $t-s=\eta$ in the outer integral, followed by a substitution $\xi-(t-\eta)=y$ in the inner integral, and then cancelling $e^{\lambda t}$, gives

$$
P(t)=-v R_{*} F_{r}^{\prime}\left(v \pi\left(R_{0}^{2}-R_{*}^{2}\right)\right) \int_{0}^{t} f_{1}(\eta) \int_{-\tau(t-\eta)}^{0} \Omega(t-\eta+y) e^{\lambda(y-\eta)} P(t-\eta+y) d y d \eta .
$$

We want to show that $\tilde{R}(t) \rightarrow 0$ as $t \rightarrow \infty$. To do so, assume, for a contradiction, that there is a $\lambda$ with $\operatorname{Re} \lambda \geq 0$. Then

$$
\begin{aligned}
|P(t)| & \leq v R_{*} F_{r}^{\prime}\left(v \pi\left(R_{0}^{2}-R_{*}^{2}\right)\right) \int_{0}^{t}\left|f_{1}(\eta)\right| \int_{-\tau(t-\eta)}^{0} \Omega(t-\eta+y) e^{(\operatorname{Re} \lambda)(y-\eta)}|P(t-\eta+y)| d y d \eta \\
& \leq v R_{*} F_{r}^{\prime}\left(v \pi\left(R_{0}^{2}-R_{*}^{2}\right)\right) \int_{0}^{\infty}\left|f_{1}(\eta)\right| \int_{-\tau(t-\eta)}^{0} \Omega(t-\eta+y)|P(t-\eta+y)| d y d \eta \\
& \leq v R_{*} F_{r}^{\prime}\left(v \pi\left(R_{0}^{2}-R_{*}^{2}\right)\right) \max _{t \in[0, T]}|P(t)| \int_{0}^{\infty}\left|f_{1}(\eta)\right| \underbrace{\left\{\int_{-\tau(t-\eta)}^{0} \Omega(t-\eta+y) d y\right\}}_{=2 \pi} d \eta .
\end{aligned}
$$

The fact that the inner integral equals $2 \pi$ follows from (2.3), after a substitution. There exists $t^{*} \in[0, T]$ such that $\left|P\left(t^{*}\right)\right|=\max _{t \in[0, T]}|P(t)|$. After evaluation of the above estimate at $t=t^{*}$, we have

$$
1 \leq 2 \pi v R_{*} F_{r}^{\prime}\left(v \pi\left(R_{0}^{2}-R_{*}^{2}\right)\right) \int_{0}^{\infty}\left|f_{1}(\eta)\right| d \eta
$$

which contradicts $(3.23)$.

In the overdamped case, when $c^{2} \geq 4 m k$, it is easy to show that $f_{1}(t) \geq 0$ for all $t \geq 0$, so that

$$
\int_{0}^{\infty}\left|f_{1}(\eta)\right| d \eta=\int_{0}^{\infty} f_{1}(\eta) d \eta=\left[\int_{0}^{\infty} f_{1}(\eta) e^{-p \eta} d \eta\right]_{p=0}=\left[\mathcal{L}\left\{f_{1}\right\}\right]_{p=0}=\frac{1}{k}
$$

by (3.24). Condition (3.23) of Theorem 3 is then the same as the second inequality of the hypotheses of Theorem 1. In the more likely scenario of underdamping, when $c^{2}<4 m k, f_{1}(t)$ decays to zero with oscillations and so $\int_{0}^{\infty}\left|f_{1}(\eta)\right| d \eta>\int_{0}^{\infty} f_{1}(\eta) d \eta=1 / k$ so that (3.23) is a stronger inequality. But recall that Theorem 3 allows variable spindle speeds, whereas Theorem 1 only applies for a constant spindle speed. 


\subsection{Effect of varying spindle speed}

Theorem 3 works for any variable (but periodic) spindle speed $\Omega(t)$ and therefore does not yield insight into the effect of varying the spindle speed. To investigate this it is desirable to introduce a new independent variable $\theta$, defined by

$$
\theta=G(t):=\int_{0}^{t} \Omega(s) d s .
$$

Since $\Omega(t)$ is the angular spindle speed, this implies that $\theta$ is the total angle through which the workpiece has turned since the start of time. This type of transformation has also been used in Otto et al [15]. It often has the effect of transforming variable delays into constant delays (the analogy in the present paper is that the lower limit on the integral in (3.31) is $\theta-2 \pi$, whereas in (2.6) it is $t-\tau(t)$ ). Similar changes of variable have been used in ecological models, in which setting the idea is usually to make the size or mass of an organism the independent variable. This can be advantageous in situations where an insect or amphibian undergoes metamorphosis on reaching a certain critical size or mass, and the time taken to achieve this state is not a constant (perhaps because the availability of food varies with the seasons). These issues were considered by Smith [20] and Brunner et al [3].

We also introduce the new dependent variable $\mathcal{R}(\theta)$, defined such that

$$
R(t)=\mathcal{R}\left(\int_{0}^{t} \Omega(s) d s\right)=\mathcal{R}(\theta) .
$$

Note that

$$
\theta=\int_{0}^{t-\tau(t)} \Omega(s) d s+\int_{t-\tau(t)}^{t} \Omega(s) d s=\int_{0}^{t-\tau(t)} \Omega(s) d s+2 \pi
$$

so that if $t$ corresponds to $\theta$ then $t-\tau(t)$ corresponds to $\theta-2 \pi$. Under the transformation the integral term in (2.6) loses the $\Omega(s)$ factor in its integrand, but $\Omega(t)$ reappears in the coefficients via the new function $\Phi(\theta)$, defined such that

$$
\Omega(t)=\Omega\left(G^{-1}(\theta)\right):=\Phi(\theta) .
$$

The transformed differential equation is

$$
\begin{aligned}
& m(\Phi(\theta))^{2} \frac{d^{2} \mathcal{R}(\theta)}{d \theta^{2}}+m \Phi(\theta) \Phi^{\prime}(\theta) \frac{d \mathcal{R}(\theta)}{d \theta}+c \Phi(\theta) \frac{d \mathcal{R}(\theta)}{d \theta}+k \mathcal{R}(\theta) \\
& =F_{r}\left(\frac{1}{2} v \int_{\theta-2 \pi}^{\theta}\left(R_{0}^{2}-\mathcal{R}^{2}(\bar{\theta})\right) d \bar{\theta}\right)-k\left(l_{0}-R_{*}\right)
\end{aligned}
$$

in which $R_{0}$ is still the original radius of the workpiece before machining commences, and $R_{*}$ is again the desired final radius which is a steady state of (3.31) satisfying (2.7). Letting $\mathcal{R}(\theta)=R_{*}+\tilde{\mathcal{R}}(\theta)$, with $\tilde{\mathcal{R}}$ small, we find that the linearisation of (3.31) about the steady state $R^{*}$ is given by

$$
\begin{aligned}
& m(\Phi(\theta))^{2} \frac{d^{2} \tilde{\mathcal{R}}(\theta)}{d \theta^{2}}+m \Phi(\theta) \Phi^{\prime}(\theta) \frac{d \tilde{\mathcal{R}}(\theta)}{d \theta}+c \Phi(\theta) \frac{d \tilde{\mathcal{R}}(\theta)}{d \theta}+k \tilde{\mathcal{R}}(\theta) \\
& =-v R_{*} F_{r}^{\prime}\left(v \pi\left(R_{0}^{2}-R_{*}^{2}\right)\right) \int_{\theta-2 \pi}^{\theta} \tilde{\mathcal{R}}(\bar{\theta}) d \bar{\theta} .
\end{aligned}
$$


We shall study (3.32) in the case when the angular spindle speed varies periodically about some constant mean speed $\Omega_{0}$, and it will be necessary to have the characteristic equation of the linearisation for the case when $\Omega(t) \equiv \Omega_{0}$. That characteristic equation, corresponding to the ansatz $\tilde{\mathcal{R}}(\theta)=\exp (\Lambda \theta)$, is

$$
m \Omega_{0}^{2} \Lambda^{2}+c \Omega_{0} \Lambda+k=-v R_{*} F_{r}^{\prime}\left(v \pi\left(R_{0}^{2}-R_{*}^{2}\right)\right)\left(\frac{1-e^{-2 \pi \Lambda}}{\Lambda}\right) .
$$

Now suppose that $\Omega_{0}$ is such that, when $\Omega(t) \equiv \Omega_{0}$, the characteristic equation (3.33) has a pair of purely imaginary roots $\Lambda= \pm i \omega_{0}$, with $\omega_{0}>0$. Then the steady state $R_{*}$ is marginally stable, and one expects to see the onset of chatter when $\Omega_{0}$ is perturbed slightly, in the right direction, from $\Omega_{0}$. Our intention is to study the potential, in this situation, for stabilising the steady state $R_{*}$ by suitably varying the spindle speed. More precisely, we make it a small amplitude periodic function of period $T$ which has mean $\Omega_{0}$, so that

$$
\Omega(t)=\Omega_{0}+\epsilon \Omega_{1}(t)
$$

where $\epsilon \geq 0$ is small $\left(\epsilon>0\right.$ for a periodic $\Omega(t)$ ), and $\Omega_{1}(t)$ is a $T$-periodic function of zero mean.

When $\epsilon=0$ the steady state $R_{*}$ is marginally stable with the onset of a periodic solution of period $2 \pi / \omega_{0}$; this determines a fast time-scale for the problem. Our expectation is that, when $\epsilon>0$, generically the steady state $R_{*}$ will either be stable or unstable but with decay or growth occurring on a slow time-scale, since $\epsilon$ is small and therefore we remain close to the stability boundary. For $\epsilon>0$ this leads us to define two time-scales

$$
T_{0}=\theta, \quad T_{1}=\epsilon \theta
$$

and to seek a solution of the linearised problem of the form

$$
\tilde{\mathcal{R}}(\theta ; \epsilon)=\tilde{\mathcal{R}}_{0}\left(T_{0}, T_{1}\right)+\epsilon \tilde{\mathcal{R}}_{1}\left(T_{0}, T_{1}\right)+\epsilon^{2} \tilde{\mathcal{R}}_{2}\left(T_{0}, T_{1}\right)+\cdots
$$

with $T_{0}$ and $T_{1}$ treated as independent variables. Note that, when $\epsilon=0$, the linearised equation (3.32) will have a solution

$$
\tilde{\mathcal{R}}(\theta ; 0)=e^{i \omega_{0} \theta}+\text { c.c. }
$$

where c.c. denotes complex conjugate. Corresponding to (3.34) we have

$$
\Phi(\theta)=\Omega_{0}+\epsilon \Phi_{1}(\theta), \quad \Phi_{1}(\theta)=\Omega_{1}\left(G^{-1}(\theta)\right) .
$$

We claim that $\Phi_{1}(\theta)$ (and hence also $\Phi(\theta)$ ) is periodic with period $G(T)$. Since $\Omega_{1}(\cdot)$ has period $T$, it suffices to show that

$$
G^{-1}(\theta+G(T))=G^{-1}(\theta)+T .
$$

But, from (3.28), $\theta=\int_{0}^{G^{-1}(\theta)} \Omega(s) d s$ and therefore

$$
\begin{aligned}
\theta+G(T) & =\int_{0}^{G^{-1}(\theta)} \Omega(s) d s+\int_{0}^{T} \Omega(s) d s \\
& =\int_{0}^{G^{-1}(\theta)} \Omega(s) d s+\int_{G^{-1}(\theta)}^{G^{-1}(\theta)+T} \Omega(s) d s \quad \text { since } \Omega(t) \text { is } T \text {-periodic } \\
& =\int_{0}^{G^{-1}(\theta)+T} \Omega(s) d s=G\left(G^{-1}(\theta)+T\right)
\end{aligned}
$$


and (3.37) follows. So $\Phi(\theta)$ is $G(T)$-periodic. However, $\Phi_{1}(\theta)$ does not have mean zero. In fact, using (3.34) and that $\int_{0}^{T} \Omega_{1}(\xi) d \xi=0$,

$$
\int_{0}^{G(T)} \Phi_{1}(\theta) d \theta=\int_{0}^{G(T)} \Omega_{1}\left(G^{-1}(\theta)\right) d \theta=\int_{0}^{T} \Omega_{1}(\xi) \Omega(\xi) d \xi=\epsilon \int_{0}^{T} \Omega_{1}^{2}(\xi) d \xi>0
$$

which will be important later.

We substitute (3.35) into the linearised equation (3.32), with $\Phi(\theta)=\Omega_{0}+\epsilon \Phi_{1}(\theta)$, requiring it to hold for all sufficiently small $\epsilon$, and compare coefficients in the usual way. Computation of the derivative terms in (3.32) is standard but the integral term is a little more delicate due to the need for the second argument of each $\tilde{\mathcal{R}}_{i}, i=1,2, \ldots$, to be $T_{1}$ which is initially treated as a parameter. Each $\tilde{\mathcal{R}}_{i}$ needs to be Taylor expanded about the point $\left(\bar{\theta}, T_{1}\right)$. Up to order $\epsilon$, the integral is computed as follows:

$$
\begin{aligned}
\int_{T_{0}-2 \pi}^{T_{0}} \tilde{\mathcal{R}}(\bar{\theta} ; \epsilon) d \bar{\theta} & =\int_{T_{0}-2 \pi}^{T_{0}}\left\{\tilde{\mathcal{R}}_{0}(\bar{\theta}, \epsilon \bar{\theta})+\epsilon \tilde{\mathcal{R}}_{1}(\bar{\theta}, \epsilon \bar{\theta})\right\} d \bar{\theta} \\
& =\int_{T_{0}-2 \pi}^{T_{0}}\left\{\tilde{\mathcal{R}}_{0}\left(\bar{\theta}, T_{1}\right)+\left(\epsilon \bar{\theta}-T_{1}\right) \frac{\partial \tilde{\mathcal{R}}_{0}}{\partial T_{1}}\left(\bar{\theta}, T_{1}\right)+\epsilon \tilde{\mathcal{R}}_{1}\left(\bar{\theta}, T_{1}\right)+O\left(\epsilon^{2}\right)\right\} d \bar{\theta} \\
& =\int_{T_{0}-2 \pi}^{T_{0}}\left\{\tilde{\mathcal{R}}_{0}\left(\bar{\theta}, T_{1}\right)+\epsilon\left(\bar{\theta}-T_{0}\right) \frac{\partial \tilde{\mathcal{R}}_{0}}{\partial T_{1}}\left(\bar{\theta}, T_{1}\right)+\epsilon \tilde{\mathcal{R}}_{1}\left(\bar{\theta}, T_{1}\right)+O\left(\epsilon^{2}\right)\right\} d \bar{\theta}
\end{aligned}
$$

Comparing coefficients of $\epsilon^{0}$, we obtain

$$
m \Omega_{0}^{2} \frac{\partial^{2} \tilde{\mathcal{R}}_{0}}{\partial T_{0}^{2}}+c \Omega_{0} \frac{\partial \tilde{\mathcal{R}}_{0}}{\partial T_{0}}+k \tilde{\mathcal{R}}_{0}+v R_{*} F_{r}^{\prime}\left(v \pi\left(R_{0}^{2}-R_{*}^{2}\right)\right) \int_{T_{0}-2 \pi}^{T_{0}} \tilde{\mathcal{R}}_{0}\left(\bar{\theta}, T_{1}\right) d \bar{\theta}=0 .
$$

By the assumption of marginal stability of the steady state $R_{*}$ in the case when $\epsilon=0$, this has solution

$$
\tilde{\mathcal{R}}_{0}\left(T_{0}, T_{1}\right)=A\left(T_{1}\right) e^{i \omega_{0} T_{0}}+\text { c.c. }
$$

for some function $A\left(T_{1}\right)$ to be determined later. Comparing coefficients of $\epsilon$,

$$
\begin{aligned}
& m \Omega_{0}^{2} \frac{\partial^{2} \tilde{\mathcal{R}}_{1}}{\partial T_{0}^{2}}+c \Omega_{0} \frac{\partial \tilde{\mathcal{R}}_{1}}{\partial T_{0}}+k \tilde{\mathcal{R}}_{1}+v R_{*} F_{r}^{\prime}\left(v \pi\left(R_{0}^{2}-R_{*}^{2}\right)\right) \int_{T_{0}-2 \pi}^{T_{0}} \tilde{\mathcal{R}}_{1}\left(\bar{\theta}, T_{1}\right) d \bar{\theta} \\
& =-2 m \Omega_{0}^{2} \frac{\partial^{2} \tilde{\mathcal{R}}_{0}}{\partial T_{0} \partial T_{1}}-2 m \Omega_{0} \Phi_{1}\left(T_{0}\right) \frac{\partial^{2} \tilde{\mathcal{R}}_{0}}{\partial T_{0}^{2}}-m \Omega_{0} \Phi_{1}^{\prime}\left(T_{0}\right) \frac{\partial \tilde{\mathcal{R}}_{0}}{\partial T_{0}}-c \Omega_{0} \frac{\partial \tilde{\mathcal{R}}_{0}}{\partial T_{1}}-c \Phi_{1}\left(T_{0}\right) \frac{\partial \tilde{\mathcal{R}}_{0}}{\partial T_{0}} \\
& -v R_{*} F_{r}^{\prime}\left(v \pi\left(R_{0}^{2}-R_{*}^{2}\right)\right) \int_{T_{0}-2 \pi}^{T_{0}}\left(\bar{\theta}-T_{0}\right) \frac{\partial \tilde{\mathcal{R}}_{0}}{\partial T_{1}}\left(\bar{\theta}, T_{1}\right) d \bar{\theta} \\
& =\left[-2 m \Omega_{0}^{2} i \omega_{0} A^{\prime}\left(T_{1}\right)+2 m \Omega_{0} \Phi_{1}\left(T_{0}\right) \omega_{0}^{2} A\left(T_{1}\right)-m \Omega_{0} \Phi_{1}^{\prime}\left(T_{0}\right) i \omega_{0} A\left(T_{1}\right)-c \Omega_{0} A^{\prime}\left(T_{1}\right)\right. \\
& \left.-c \Phi_{1}\left(T_{0}\right) i \omega_{0} A\left(T_{1}\right)-v R_{*} F_{r}^{\prime}\left(v \pi\left(R_{0}^{2}-R_{*}^{2}\right)\right)\left\{\frac{2 \pi}{i \omega_{0}} e^{-2 \pi i \omega_{0}}+\frac{1}{\omega_{0}^{2}}\left(1-e^{-2 \pi i \omega_{0}}\right)\right\} A^{\prime}\left(T_{1}\right)\right] e^{i \omega_{0} T_{0}} \\
& + \text { c.c. }
\end{aligned}
$$


after inserting (3.40) and evaluating the integral. We want the solution $\tilde{\mathcal{R}}_{1}\left(T_{0}, T_{1}\right)$ of this equation to be bounded as a function of $T_{0}$. The fact that the right hand side of (3.41) is multiplied by $e^{i \omega_{0} T_{0}}$, which is precisely the solution of the corresponding homogeneous equation (3.39), raises the possibility that the solution $\tilde{\mathcal{R}}_{1}\left(T_{0}, T_{1}\right)$ of $(3.41)$ could contain secular terms. However, the square bracketed coefficient of $e^{i \omega_{0} T_{0}}$ in the right hand side of (3.41) is a periodic function of $T_{0}$, which could be expanded as a Fourier series. If that Fourier series contained a constant term, this would be multiplied by $e^{i \omega_{0} T_{0}}$ and secular terms would arise. Therefore, the way to suppress secular terms is to require the constant term of the Fourier series to be zero. In other words, the mean of the square bracketed term in (3.41) over one period, and therefore also its integral with respect to $T_{0}$ over the interval $T_{0} \in[0, G(T)]$, should be zero. This gives

$$
\begin{aligned}
& \underbrace{\left[2 m \Omega_{0}^{2} i \omega_{0}+c \Omega_{0}+v R_{*} F_{r}^{\prime}\left(v \pi\left(R_{0}^{2}-R_{*}^{2}\right)\right)\left\{\frac{2 \pi}{i \omega_{0}} e^{-2 \pi i \omega_{0}}+\frac{1}{\omega_{0}^{2}}\left(1-e^{-2 \pi i \omega_{0}}\right)\right\}\right]}_{Z} G(T) A^{\prime}\left(T_{1}\right) \\
& =\left(2 m \Omega_{0} \omega_{0}^{2}-c i \omega_{0}\right) A\left(T_{1}\right) \int_{0}^{G(T)} \Phi_{1}\left(T_{0}\right) d T_{0} \\
& =\left(2 m \Omega_{0} \omega_{0}^{2}-c i \omega_{0}\right) A\left(T_{1}\right) \epsilon \int_{0}^{T} \Omega_{1}^{2}(\xi) d \xi .
\end{aligned}
$$

This is an ordinary differential equation to be solved for $A\left(T_{1}\right)$, and routine computations show that if

$$
2 m \Omega_{0} \omega_{0}^{2} \operatorname{Re} Z-c \omega_{0} \operatorname{Im} Z<0,
$$

where $Z$ is the large square bracketed term in (3.42), then $A\left(T_{1}\right) \rightarrow 0$ as $T_{1} \rightarrow \infty$. Now, $\Lambda=i \omega_{0}$ satisfies the characteristic equation (3.33), so that

$$
-m \Omega_{0}^{2} \omega_{0}^{2}+c \Omega_{0} i \omega_{0}+k=-v R_{*} F_{r}^{\prime}\left(v \pi\left(R_{0}^{2}-R_{*}^{2}\right)\right)\left(\frac{1-e^{-2 \pi i \omega_{0}}}{i \omega_{0}}\right) .
$$

Comparing real and imaginary parts gives

$$
\begin{array}{r}
c \Omega_{0} \omega_{0}^{2}=v R_{*} F_{r}^{\prime}\left(v \pi\left(R_{0}^{2}-R_{*}^{2}\right)\right)\left(1-\cos 2 \pi \omega_{0}\right), \\
\omega_{0}\left(m \Omega_{0}^{2} \omega_{0}^{2}-k\right)=v R_{*} F_{r}^{\prime}\left(v \pi\left(R_{0}^{2}-R_{*}^{2}\right)\right) \sin 2 \pi \omega_{0}
\end{array}
$$

and, on eliminating the sine and cosine, we find that $\omega_{0}$ satisfies the following equation, a quadratic in $\omega_{0}^{2}$ :

$$
m^{2} \Omega_{0}^{4} \omega_{0}^{4}+\left(c^{2}-2 k m\right) \Omega_{0}^{2} \omega_{0}^{2}+k^{2}-2 c \Omega_{0} v R_{*} F_{r}^{\prime}\left(v \pi\left(R_{0}^{2}-R_{*}^{2}\right)\right)=0 .
$$

If we now solve (3.44) for $e^{-2 \pi i \omega_{0}}$, and insert the result into $Z$, we obtain $Z$ as a rational function of $\omega_{0}$. With $Z$ written in that form, the stability condition (3.43) becomes

$$
2 \pi c v R_{*} F_{r}^{\prime}\left(v \pi\left(R_{0}^{2}-R_{*}^{2}\right)\right)<4 \pi m^{2} \Omega_{0}^{3} \omega_{0}^{4}+\left(2 \pi c^{2}-4 \pi m k-m c \Omega_{0}\right) \Omega_{0} \omega_{0}^{2}-k c .
$$

In summary, we have proved the following theorem. 
Theorem 4. Suppose the angular spindle speed $\Omega(t)$ varies as described by (3.34), with $\Omega_{1}(t)$ periodic and of mean zero, and suppose parameter values are such that, when $\epsilon=$ 0 , the steady state $R(t)=R_{*}$ of (2.6) is marginally stable with $\omega_{0}$ given by (3.46). Then, if $\epsilon>0$ is sufficiently small, that steady state becomes asymptotically stable if inequality (3.47) holds.

A parameter domain in which the conditions of Theorem 4 hold is that which corresponds to the onset of high frequency chatter. Recall that, for constant spindle speeds, $\omega_{0}$ is the frequency of the periodic solution at bifurcation when $\theta$ is the independent variable. When $t$ is the independent variable the frequency becomes $\Omega_{0} \omega_{0}$. We therefore define $\omega=\Omega_{0} \omega_{0}$. We also introduce $A=v R_{*} F_{r}^{\prime}\left(v \pi\left(R_{0}^{2}-R_{*}^{2}\right)\right)$ and treat $A$ as a bifurcation parameter. For constant spindle speeds bifurcation to chatter (i.e. to periodic solutions) occurs as we cross the curve $\left\{\left(\Omega_{0}(\omega), A(\omega)\right) \in \mathbb{R}^{2}: \omega \in(\sqrt{k / m}, \infty)\right\}$ in the $\left(\Omega_{0}, A\right)$ parameter plane, where

$$
\Omega_{0}(\omega)=\pi \omega / \tan ^{-1} \frac{c \omega}{m \omega^{2}-k}, \quad A(\omega)=\frac{m^{2} \omega^{4}+\left(c^{2}-2 k m\right) \omega^{2}+k^{2}}{2 c \Omega_{0}(\omega)} .
$$

In (3.48) the expression for $A(\omega)$ comes from (3.46); that for $\Omega_{0}(\omega)$ comes from dividing the equations in (3.45). For large values of $\omega$ on this curve, we have

$$
\Omega_{0}(\omega) \sim \frac{m \pi \omega^{2}}{c}, \quad A(\omega) \sim \frac{m \omega^{2}}{2 \pi} .
$$

On replacing $\omega_{0}$ by $\omega / \Omega_{0}$ and evaluating the stability condition (3.47) along the bifurcation curve, it can be written in the form

$$
2 \pi c A(\omega)<4 \pi m^{2} \frac{\omega^{4}}{\Omega_{0}(\omega)}+\left(2 \pi c^{2}-4 \pi m k-m c \Omega_{0}(\omega)\right) \frac{\omega^{2}}{\Omega_{0}(\omega)}-k c .
$$

For large $\omega$, using (3.49), this becomes $c m \omega^{2}<3 c m \omega^{2}$. Therefore, (3.50) holds for sufficiently large $\omega$. Thus, we have shown that varying the angular spindle speed has the potential to cure high frequency chatter.

\section{Numerical simulations}

Numerical simulations confirm the analytical results of the paper, and the results of a few of these are shown in Figs. 2-4. For cases in which the spindle speed is varying (i.e., spindle speed variation SSV is on) we took $\Omega(t)$ to be sinusoidally varying about some mean $\Omega_{0}$ :

$$
\Omega(t)=\Omega_{0}+\alpha \sin \zeta t
$$

which, when $\alpha>0$, corresponds to the manner in which spindle speed can be made to vary in some real lathes. The operator enters the desired values of the mean speed $\Omega_{0}$, variation $\alpha$ and frequency $\zeta$ using a control pad. A typical SSV scenario might use a mean spindle speed of $1000 \mathrm{rpm}$ (about 104 radians per second), varying between 700 and $1300 \mathrm{rpm}$, with $\zeta=5.235$ (so that the period of the SSV cycle is about 1.2 seconds). 
For these particular values, $\Omega(t)=104+30 \sin 5.235 t$. A typical value for the natural frequency of the assembly is of the order $1500 \mathrm{~Hz}$, so that $\sqrt{k / m}=1500$. The modelling of the rigidity of the assembly requires the use of a large value for the spring constant $k$; we took $k=50,000 \mathrm{~N} / \mathrm{m}$, and so we take $m=0.02 \mathrm{~kg}$.

The numerical simulations are of the solutions of the linearised equation for $\tilde{R}(t)$; equation (3.8), with $\Omega(t)$ given by (4.51) and $\tau(t)$ by (2.3). The simulations were conducted by first converting the second order differential equation to two first order equations and then solving them numerically using the modified Euler's Method. The time for one revolution, $\tau(t)$, was found from the threshold condition (2.3), with $\Omega(t)$ given by (4.51), and the integral in (3.8) was numerically computed as a Riemann sum. These procedures were all implemented using Matlab.

For convenience we treat the coefficient of the integral term as a single parameter $A$, so that $A=v R_{*} F_{r}^{\prime}\left(v \pi\left(R_{0}^{2}-R_{*}^{2}\right)\right)$. This parameter is important as it depends strongly on the feed rate $v$. Fig. 2 shows a scenario in which SSV is off, so that $\alpha=0$ and the spindle speed is a constant $\Omega_{0}=104$ radians per second. The values for the other parameters are shown in the caption. The equation being linear, solutions generally either grow or decay but the chosen parameter values are on the boundary between these outcomes. The figure shows a sustained oscillation (a periodic solution). Note that $\tau(t)$ is constant here, since $\Omega(t)$ is constant. Fig. 3 shows a simulation of the same scenario but with SSV switched on with an amplitude $\alpha=5$, showing that this is sufficient to cure chatter. For Fig. 4 the tool feed rate $v$ as been increased; this corresponds to the use of a higher value for $A$. It turns out in this situation that SSV, even with an amplitude of $\alpha=30$, is not sufficient to cure chatter.

\section{Conclusion}

This paper rigorously derives an integro-differential equation as a model for a turning process involving a cut in which the feed is parallel to the spindle axis but the vibrations are perpendicular to the axis. Additionally, we implement spindle speed variation (SSV), allowing the spindle speed to vary in a prescribed manner. As noted earlier, there are already many papers that mathematically model SSV in various experimental scenarios in turning or milling, although many of them give rise to the same or very similar looking delay equations having the appearance of (1.1), with $\tau$ varying with time in the presence of SSV. The present paper is certainly not the first to consider the case in which cutting occurs parallel to the spindle axis, as opposed to a facing process in which the cutting is directed towards and perpendicular to that axis. Although that distinction is important, of equal importance to the modelling is the assumed direction of the vibrations. Wahi and Chatterjee [24] considered vibrations parallel to the spindle axis and tool feed direction. In contrast, in the present paper, we have assumed that the tool moves at a fixed speed $v$ in the direction of the spindle axis, with all vibration occurring in a direction perpendicular to that axis (i.e. in the $R$ direction, in our notation). Of course, vibrations in real machining operations will not involve only one degree of freedom: they may involve both the longitudinal and radial directions. However, in parallel turning, vibrations in the radial direction are especially significant in giving rise to chatter (Stone [21], pg. 38) and 

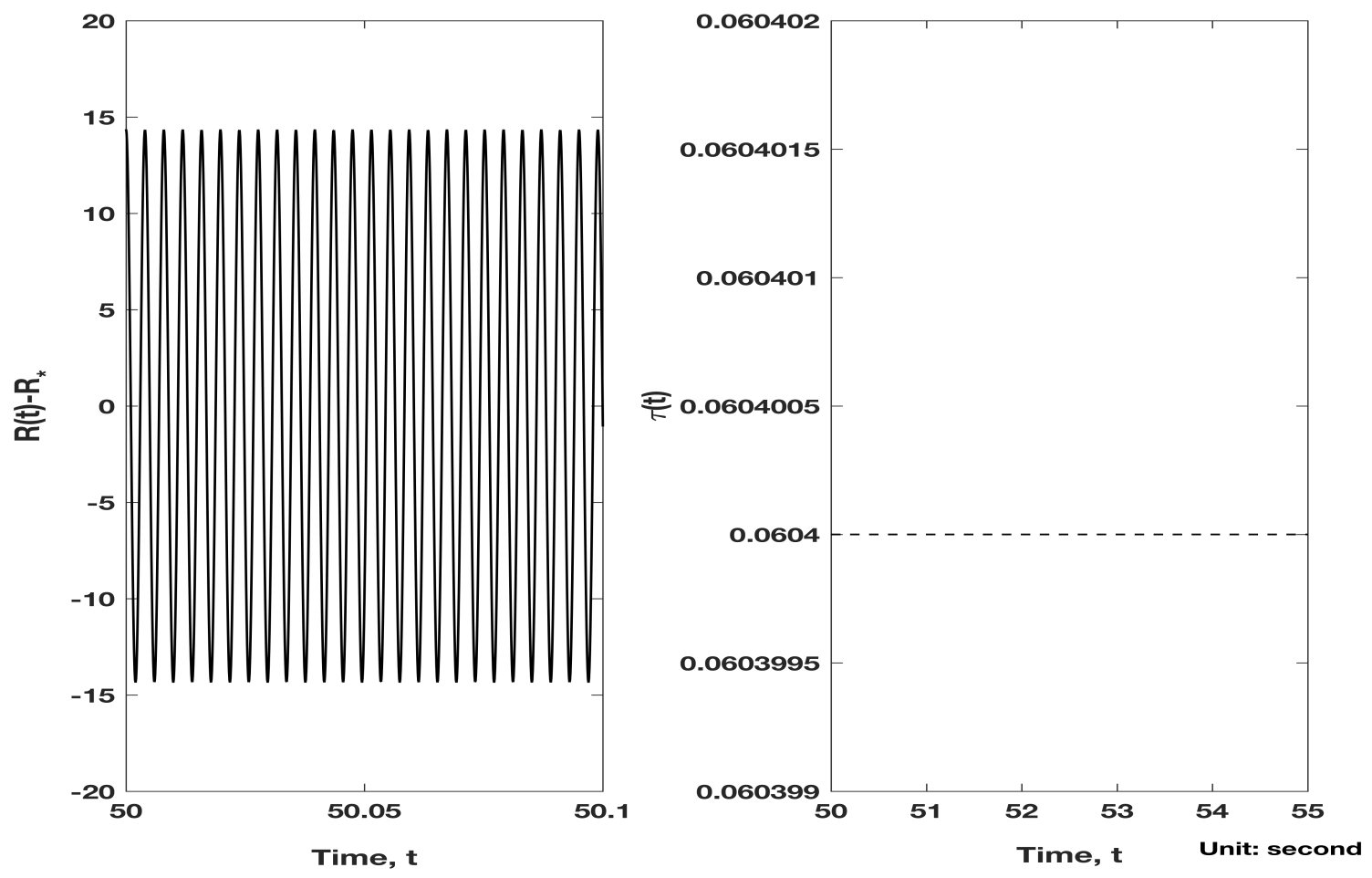

Figure 2: Situation with no SSV (spindle speed variation). Spindle speed is a constant $\Omega_{0}=104 \mathrm{rad} / \mathrm{sec}, \alpha=0$ and $\zeta=5.235$. Other parameter values were $m=0.02 \mathrm{~kg}$, $c=0.2 \mathrm{~kg} / \mathrm{sec}, k=50,000 \mathrm{~N} / \mathrm{m}$ and $A=4293.35$, where $A=v R_{*} F_{r}^{\prime}\left(v \pi\left(R_{0}^{2}-R_{*}^{2}\right)\right)$. With these values the zero solution of (3.8) is marginally stable and we see a sustained oscillation (the onset of chatter). 

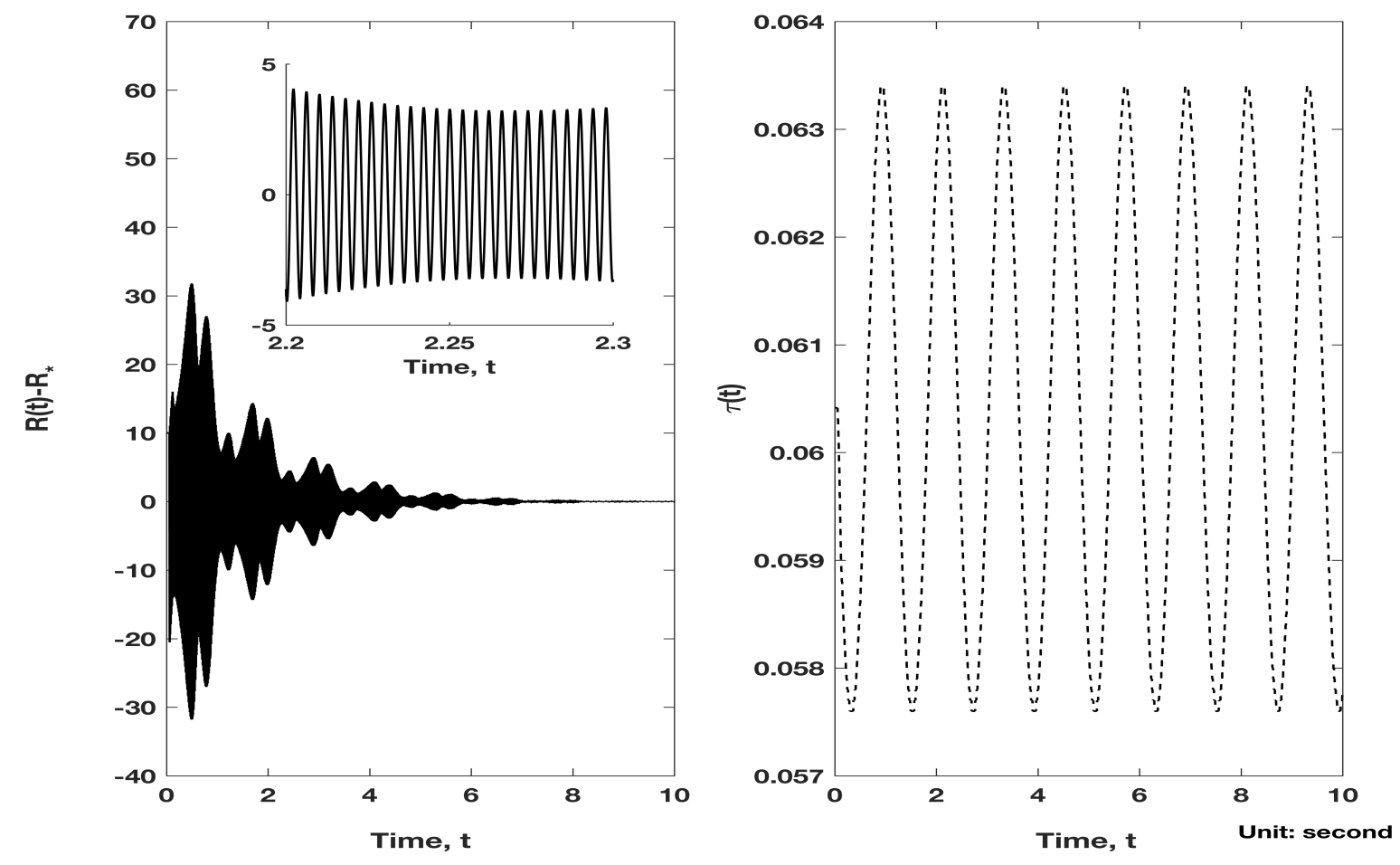

Figure 3: Parameter values for this simulation are the same as in the caption to Fig. 2, except that $\alpha=5$ here, so SSV has been switched on. The effect is that the zero solution of (3.8) has become asymptotically stable, so that chatter is eliminated. The variable $\tilde{R}(t)$ tends to zero in a manner best described as a high frequency oscillation with an amplitude that decays to zero in a non-monotone manner involving periods of growth and periods of decay. The inset to the left panel shows some of the detail of the oscillation over a short time interval. The right panel, which shows $\tau(t)$, reveals that the growth and decay of the amplitude of $\tilde{R}(t)$ occurs in time intervals that correspond to fluctuations in $\tau(t)$ (and hence in the spindle speed $\Omega(t)$ ). 

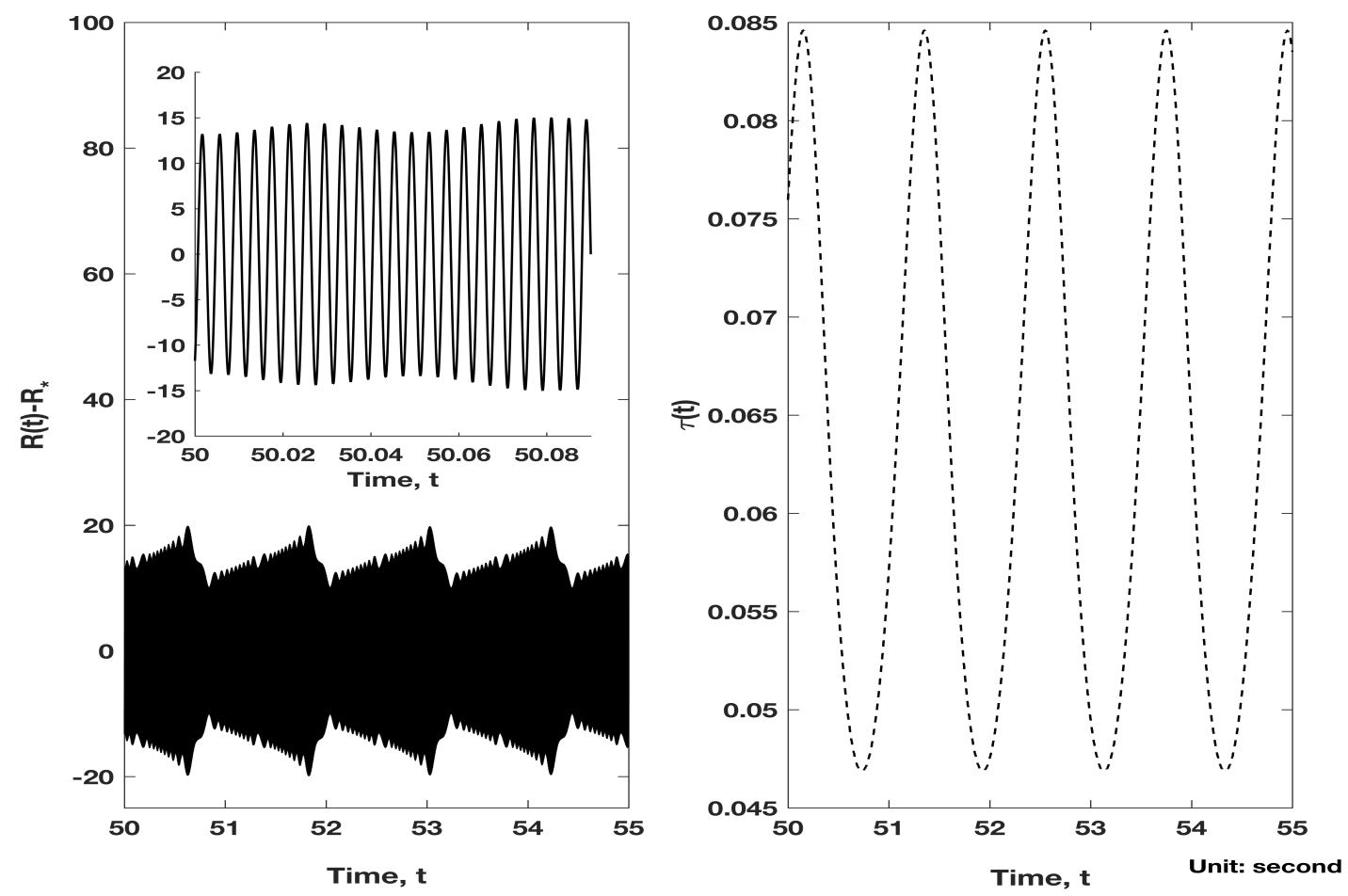

Figure 4: Situation in which spindle speed variation SSV does not cure chatter. Parameter values: $\Omega_{0}=104 \mathrm{rad} / \mathrm{sec}, \alpha=30, \zeta=5.235, m=0.02 \mathrm{~kg}, c=0.2 \mathrm{~kg} / \mathrm{sec}$, $k=50,000 \mathrm{~N} / \mathrm{m}$ and $A=5307.93$, where $A=v R_{*} F_{r}^{\prime}\left(v \pi\left(R_{0}^{2}-R_{*}^{2}\right)\right)$. Note that this simulation has used a higher value for $A$ (corresponding to a higher tool feed rate), and demonstrates that even the use of SSV with a relatively high amplitude of $\alpha=30$ does not cure chatter. Here, $\tilde{R}(t)$ takes the form of a fine scale oscillation with an amplitude that fluctuates on the same time scale as does $\tau(t)$. 
this is precisely the situation we have been concerned with in this paper.

The analysis and simulations in this paper indicate that spindle speed variation has the potential to cure high frequency chatter. Whether it actually does so or not depends to a significant extent on the tool feed rate $v$. Our work indicates that $v$ should not be too high, if SSV is to work as a chatter elimination measure.

We reiterate that in this paper we assume that the workpiece is rigid and the tool is flexible. It is well known that chatter is more likely with long, thin workpieces. If such a workpiece is unsupported with a tailstock, it may in practice bend away from the tool. Otto et al [14] have modelled this tendency using well established theory for the bending of beams, using a fourth order partial differential equation involving parameters such as the modulus of elasticity. Flexibility of both the tool and workpiece leads to increased numbers of degrees of freedom. It turns out that the effect of the mode interaction between the tool and workpiece can be counterintuitive (such as destabilisation for an increased workpiece stiffness). Khasawneh and Otto [9] present a comparable study for a process involving the use of a round insert (a scenario in which the chip thickness varies along the cutting edge).

SSV has some limitations. In practice, it requires the spindle speed to be varied on a fast time scale, i.e. the frequency $\zeta$ in expression (4.51) should be on the high side. We chose a value for $\zeta$ so that the period of the SSV cycle would be about 1.2 seconds. This is typical of real turning operations using SSV, but it is short when one considers the high inertia of the spindle, necessitating large amounts of energy to give it the required rapid acceleration and deceleration. Drawbacks of SSV therefore include increased energy costs and the potential for instability of the spindle system (Siddhpura and Paurobally [19]). Recently, it has also been suggested that the technique may shorten tool life (Albertelli et al [1]).

Acknowledgement. S.A. Gourley would like to thank the National Center for Theoretical Sciences (NCTS), Taiwan, for hosting his visit during Summer 2017. F.-B. Wang was supported in part by the Ministry of Science and Technology of Taiwan, the National Center for Theoretical Sciences (NCTS), National Taiwan University and Chang Gung Memorial Hospital (CRRPD3H0011, BMRPD18 and NMRPD5F0543). Y. Xiao thanks the Simons Foundation for its support.

\section{References}

[1] P. AlBERTELli, V. MUSSI \& M. MONNO, The analysis of tool life and wear mechanisms in spindle speed variation machining, Int. J. Adv. Manuf. Technol. 72 (2014), pp. 1051-1061.

[2] R. BELLMAN \& K.L. COOKE, Differential-difference equations, Academic Press, New York-London, 1963.

[3] H. BRUNNER, S.A. GOURLEY, R. LIU \& Y. XIAO, Pauses of larval development and their consequences for stage-structured populations, SIAM J. Appl. Math. 77 (2017), pp. 977-994. 
[4] J.M. CUSHING, Integrodifferential equations and delay models in population dynamics, Lecture Notes in Biomathematics, Vol. 20. Springer-Verlag, Berlin-New York, 1977

[5] T. ERNEUX, Applied delay differential equations, Surveys and Tutorials in the Applied Mathematical Sciences, Vol. 3. Springer, New York, 2009.

[6] C. HÖS \& A.R. CHAMPNEYS, Grazing bifurcations and chatter in a pressure relief valve model, Phys. D. 241 (2012), pp. 2068-2076.

[7] T. INSPERGER \& G. STEPAN, Stability analysis of turning with periodic spindle speed modulation via semidiscretization, J. Vibr. Control 10 (2004), pp. 1835-1855.

[8] T. KALMÁR-NAGY, G. STÉPÁN \& F.C. MOON, Subcritical Hopf bifurcation in the delay equation model for machine tool vibrations, Nonlinear Dynam. 26 (2001), pp. 121-142.

[9] F.A. KHASAWNEH \& A. OTTO, Effect of the interaction between tool and workpiece modes on turning with round inserts, Int. J. Dynamics and Control 6 (2018), pp. $571-581$.

[10] Y. KUANG, Delay differential equations with applications in population dynamics, Mathematics in Science and Engineering, 191. Academic Press, Inc., Boston, MA, 1993.

[11] M.M. NIGM, A method for the analysis of machine tool chatter, Int. J. Machine Tool Design and Research. 21 (1981), pp. 251-261.

[12] A. OTTO, G. KEHL, M. MAYER \& G. RADONS, Stability Analysis of Machining with Spindle Speed Variation. In Modelling of Machining Operations. Book Series: Advanced Materials Research Vol. 223 (2011).

[13] A. OTTO \& G. RADONS, Application of spindle speed variation for chatter suppression in turning, CIRP J. Manufacturing Science and Technology 6 (2013), pp. $102-109$.

[14] A. OTTO, F.A. KHASAWNEH \& G. RADONS, Position-dependent stability analysis of turning with tool and workpiece compliance, Int. J. Advanced Manufacturing Technology. 79 (2015), pp. 1453-1463.

[15] A. OTTO \& G. RADONS, Transformations from Variable Delays to Constant Delays with Applications in Engineering and Biology. In: Insperger T., Ersal T., Orosz G. (eds) Time Delay Systems. Advances in Delays and Dynamics, Vol 7. Springer, Cham (2017).

[16] S.S. PARK \& Y.M. QIN, Robust regenerative chatter stability in machine tools, Int. J. Advanced Manufacturing Technology. 33 (2007), pp. 389-402. 
[17] S. SEGUY, T. INSPERGER, L. ARNAUD, G. DESSEIN \& G. PEIGNÉ, On the stability of high-speed milling with spindle speed variation, Int. J. Adv. Manuf. Technol 48 (2010), pp. 883-895. https://doi.org/10.1007/s00170-009-2336-9

[18] H.M. SHI \& S.A. TOBIAS, Theory of finite amplitude machine tool instability, Int. J. Machine Tool Design and Research 24 (1984), pp. 45-69.

[19] M. SIDDHPURA \& R. PAUROBALLY, A review of chatter vibration research in turning, Int. J. Machine Tools and Manufacture 61 (2012), pp. 27-47.

[20] H.L. SMITH, Reduction of structured population models to threshold-type delay equations and functional-differential equations: a case study, Math. Biosci. 113 (1993), pp. 1-23.

[21] B. STONE, Chatter and Machine Tools, Springer Cham, Heidelberg, New York, Dordrecht, London (2014).

[22] N. SRI NAMACHCHIVAYA \& R. BEDDINI, Spindle speed variation for the suppression of regenerative chatter, J. Nonlinear Sci. 13 (2003), pp. 265-288.

[23] S.A. TOBIAS, Machine-tool vibration, Wiley, 1965.

[24] P. WAHI \& A. CHATTERJEE, Regenerative Tool Chatter Near a Codimension 2 Hopf Point Using Multiple Scales, Nonlinear Dyn 40 (2005), pp. 323-338.

[25] J. WU, Theory and applications of partial functional-differential equations, Applied Mathematical Sciences, 119. Springer-Verlag, New York, 1996.

[26] H. ZHANG, M.J. JACKSON \& J. NI, Stability analysis on spindle speed variation method for machining chatter suppression, Int. J. Machining and Machinability of Materials 5 (2009), pp. 107-128.

[27] H. ZHANG, M.J. JACKSON \& J. NI, Spindle speed variation method for regenerative machining chatter control, Int. J. of Nanomanufacturing 3 (2009), pp. 73-99. 